\title{
A Context Model for Intelligible Explanations in Adaptive Personalized Learning Environments
}

\author{
Mandy Goram and Dirk Veiel
}

\begin{abstract}
Supporting user friendly intelligible and comprehensive explanations in context-based, adaptive systems is a big challenge. They are important for a personalized system to support user acceptance and user trust. In cases, where privacy laws like the General Data Protection Regulation (GDPR) are affected, it's even more challenging. GDPR e. g. demands explanations of data usages, $i$. e. explanations where and for what purpose personal data is being processed. Currently, users cannot retrace the usage and the storage of their personal data in context-based adaptive collaboration environments. We address the aforementioned problem by developing a context-based adaptive platform linked to an adaptive personalized learning environment (APLE) to support learners with intelligible, comprehensible explanations of system processes.
\end{abstract}

Index Terms-Comprehensibility, context-based adaptive systems, context modelling, GDPR, intelligibility, ontology, personal explanations, privacy law.

\section{INTRODUCTION}

The educational landscape has changed tremendously in recent years. Distance learning has been established worldwide and offers people of different backgrounds and personal circumstances a wide range of educational programs. In addition, technological changes that are used in developing modern e-learning platforms require life-long learning. In many cases, these e-learning platforms are based on a one-fits-all concept that cannot meet learners needs. Personalization and adaptation may help by taking individual skills, backgrounds and circumstances of the related learner into account.

According to [1], personalization means to adapt the learning environment or the content accordingly to the learner and her/his situation. Adaptive learning is "the capability to modify any individual student's learning experience as a function of information obtained through their performance on situated tasks or assessments" [1].

Adaptive learning supports learners to develop problem solving strategies by guidance from predefined rules [2]. It is used to apply information filtering to "provide only relevant and categorized outputs to the user" [2].

Adapting learning paths through the course material in different ways and changing the content from learning

\footnotetext{
Manuscript received July 15, 2019; revised March 9, 2020. This work was supported by the Research Cluster Digitalization, Diversity and Lifelong Learning. Consequences for Higher Education $\left(D^{2} L^{2}\right)$ of the FernUniversität in Hagen funded by the Ministry of Culture and Science of the German State of North Rhine-Westphalia.

The authors are with the FernUniversität in Hagen, Faculty of Mathematics and Computer Science, 58084 Hagen, Germany (e-mail: mandy.goram@fernuni-hagen.de,dirk.veiel@fernuni-hagen.de).
}

activities [2] are two examples of possible adaptations in personalized learning environments. Furthermore, adaptation can be used to support collaborative learning situations of student groups, e. g. to organize or to create suitable student groups [2].

Adaptation in learning environments is used to create a learner experience [3] respecting the individual learners background, knowledge and conditions of learning with the intention to increase the learning success. An adaptive learning system requires functionalities to interact with the learning design, the content and the learners [2], [3].

To support learners in certain situations (e. g. developing problem solving strategies) the system must be aware of the learner's situation and the surrounding socio-technical environment, i. e. the context. Dey [4] defines that "Context is any information that can be used to characterize the situation of an entity". Considering the users context, a system becomes context-aware, as soon as it "uses context to provide relevant information and/or services to the user, where relevancy depends on the user's task" [4]. The disadvantage of context-aware systems is the predefined and fixed context to a certain domain and some few situations [5]. Because it is not possible to define all possible situations of the users and their interactions a priori, the system is limited in supporting learners. Providing an explicit context representation of a system at runtime adds flexibility to supporting users in related situations of specific domains [6].

\section{A. Problem Statement}

According to [7], supporting user friendly intelligible and comprehensive explanations in context-based, adaptive systems is a big challenge. They are important for a personalized system to support user acceptance and user trust. In cases, where privacy laws like the General Data Protection Regulation (GDPR) are affected, it's even more challenging. GDPR e. g. demands explanations of data usages, i. e. explanations where and for what purpose personal data is being processed. Currently, users cannot retrace the usage and the storage of their personal data in context-based adaptive collaboration environments.

\section{B. Approach}

We address the aforementioned problem by developing a context-based adaptive platform linked to an adaptive personalized learning environment (APLE) which should support comprehensibility and intelligibility. To facilitate the comprehensibility in that kind of systems we extend the domain model of the context-based adaptive collaborative platform CONTact [6]. In this paper we propose a domain model for intelligible explanations for learning environments. 
In Section II we present related work of personalized learning environments and discuss, how they address the aforementioned problem statement. We illustrate our context model and the related approach to ensure comprehensibility of APLE in Section III, before we discuss the domain model in more detail in Section IV. Finally, we present some conclusions (Section V) and future work (Section VI).

\section{RELATED WORK}

For several years, scientists worked on designing personalized, adaptive learning environments. In their research, they concentrated on representing pedagogical and learner-centered aspects. Some of them took context information into account and represented it as an ontology [5] An ontology is a formal specification of a certain domain which describes a set of concepts, relationships and formal axioms that restrict the interpretation of concept instances [8]. The formal concepts can become a common ground to describe a specific domain which can be shared and reused.

[9] present an adaptive personalized e-learning architecture. It is based on a course ontology containing concepts about learning objects and a user ontology containing learner details and learning styles. The ontologies are limited to course and user. [10] focus on self-regulated learning in an adaptive learning system. They describe user models and profiles but no formal representation.

The context-aware learning system in [11] has a domain model that is used in a multi-agent system approach to support learners in different situations. The domain model contains course topics, learning sequences regarding a course topic and learner models. The domain model of the multi-agent-based mobile learning system of [12] represents the knowledge of the learning area, information about possible didactical solutions and specific information for individual users to personalize the learning process. Both systems have a predefined and fixed context. This makes it impossible to use it to other learning systems.

The context-based adaptive system approach of [5] decouple the context from the content as much as possible. It can be reused and modified to context changes. They extend the Learning Objects Metadata (LOM) standard by enriching content context. That makes it possible to compose, reuse and adapt educative content provided by third parties. [5] present a combination of three context models in a multi-agent-based e-Learning platform.

[13] present a generic four-layer framework for modelling context in a collaboration environment, a generic adaptation process, and a collaboration domain model for describing collaboration environments and collaboration situations. [6] implements the framework, using an extended domain model and the related adaptation process. The resulting CONTact platform is able to sense and formalize users' interaction with the system at runtime, and to adapt according to the user's current collaboration situation. These adaptations may confuse users. Therefore, [14] use context enriched explanations to help users understand the adaptation behavior.

All of the aforementioned approaches are able to offer a personalized adaptation of content or support the learner in specific situations by using either fixed rules for special situations or a formal model. Except for [6] and [14], none is concerned with the aspects of the comprehensibility of system behavior, decisions and data processing. [6] and [14] take this aspect into account, but do not satisfy legal requirements. So far, there are no known context-based e-Learning systems that support comprehensibility and intelligibility for learners. There are also no approaches that would meet the requirements of the privacy law GDPR.

\section{CONTEXT ModELING}

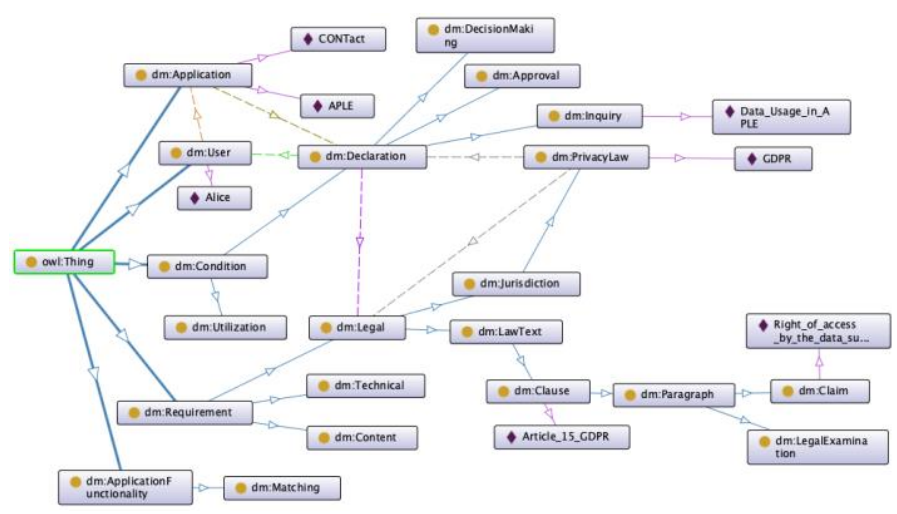

Fig. 1. Ontology representing legal and comprehensibility concepts and relations.

In this section we present our domain model and its concepts and relationships are introduced. We use the Web Ontology Language (OWL) and the Protégé Ontology Editor [15] for context modelling.

In this paper we focus on comprehensibility and intelligibility and do not consider a pedagogical, knowledge or learner perspective which are commonly used for context-aware or context-based learning environments [5], [11]. These are part of a separate domain model which could be integrated into our domain model. We use our generic four-layer framework (i. e. domain model, state, contextualized state and adapted state) for modelling context in a collaboration environment and extend the related collaboration domain model for describing collaboration environments and collaboration situations [13] to illustrate our approach.

\section{A. Overview}

Fig. 1 shows our ontology. It helps describing the situation when comprehensibility and intelligibility is needed. Therefore, we illustrate the approach with the student Alice, who wants to get an overview of her stored and processed (personalized) data. Fig. 1 shows Alice an instance of the concept $d m:$ User. She interacts with the APLE environment. For readability reasons, we left some concepts and relationships between concepts, and focused on the aspects of comprehensibility and legal requirements. APLE and CONTact are instances of the concept $d m$ :Application. Figure 1 shows the dependencies of the concepts $d m$ :Condition, dm:Requirement and dm:Declaration.

Conditions determine what an application (dm:Application) or an application functionality (dm:ApplicationFunctionality) has to consider at runtime and 
how it should deal with certain situations. The concept contains a set of rules with the form If... Then... Else. The set of rules describes the situations in which a certain action is expected. During runtime, it checks the actions of an application and look for rules that have to be considered.

Requirements are defined in the concept dm:Requirement. The set of rules for the conditions is derived from these requirements. The requirements are checked at runtime and are not stored as a fixed set of rules. Requirements can be related to technical conditions (dm:Technical), aspects of content (dm:Content) (e.g. the specification of a lecturer which learning plan is assigned to a learning type) and/or legal regulations ( $\mathrm{dm}$ :Legal). The design of the three factors are independent concepts that are described in separate domain models. Figure 1 shows parts of the domain model for the legal regulations (dm:Legal). These will be discussed in more detail in the following section.

The concept dm:Declaration is used to provide comprehensible explanations. As Fig. 1 shows, the provision of an explanation depends on the requirements (e. g. legal regulations). The context is used to determine what has to be explained and how it has to be made available to the user. The declaration is composed by the conditions and requirements. These are linked and formatted at runtime when the system creates an explanation.

\section{B. Legal Context Ontology}

The domain model in Fig. 1 contains concepts to describe the German jurisdictions by depicting their taxonomy as part of the concept dm:Jurisdiction. Every legal area of the taxonomy could contain its own domain model with specific concepts. Next to the German jurisdiction it is also possible to extend the domain model with taxonomies of other jurisdictions.

The individual legal texts are not part of the model, because they are subject to regular changes. This would lead to constant adjustments of the model. Therefore, the required legal regulations are instantiated at runtime when certain conditions are meet. For readability reasons, Fig. 1 only contains the privacy law (dm:PrivacyLaw). The instance $G D P R$ is the specific law.

The domain model also depicts the general structure of the legal texts with the concept $d m$ :LawText including its clauses (dm:Clause) and paragraphs (dm:Paragraph). A paragraph can either represent a claim (dm:Claims) and or a legal explanation (dm:LegalExplanation). Fig. 1 shows the instance Right_of_access_by_the_data_subject of dm:Claim. This taxonomy makes it possible to link legal regulations to a processable structure.

The legal concepts of the domain model are important to support intelligible legal explanations through the system. Experts in the field of law shall be responsible for depositing intelligible declarations for the law and their linking to the legal domain models structure. The resulting templates could be used to provide explanations at runtime by creating instances of the concept dm:Declaration.

\section{Comprehensibility Context Ontology}

As mentioned before, external factors such as legal requirements could determine the processes and functionalities of a system. These requirements should be transparent to support the comprehensibility.

The concept $d m$ :Declaration of the domain model (Figure 1) makes it possible to create supportive declarations about the processing and conditions of the system to the users. The concept of declarations is related to the conditions (dm:Condition). The conditions in Fig. 1 are derived from the legal requirements (dm:Legal) and must be taken into account by the system at runtime.

Next to the provision of explanations for users the use of certain functionalities of the system maybe also motivated from a legal perspective. That may not need interaction with the user. An example of this can be the requirement for encrypted data transmission, which is integrated in the domain model by the concept dm:Utilization.

In case of legal requirements, the conditions result from the legal regulations of the affected jurisdictions. Fig. 1 contains the instance Article_15_GDPR of the concept $d m$ :Clause representing the requirements of Article 15 of the GDPR (https://gdpr-info.eu/art-15-gdpr/). According to Article 15 of the GDPR, data subjects whose data are collected and processed have a right to obtain information about the usage. Access shall include the purposes of the processing, the categories of personal data processed, the recipients to whom the data are disclosed, the duration of the storage, the existence of a right of appeal and an overview of the origin of the data, if not collected from the data subject. The domain model consider that kind of regulation and its condition to the declaration with the concept dm:Inquiry. The instance Data_Usage_in_APLE represents an explanation about the data usage in $A P L E$.

In addition, Article 15 declares, the data subject has the right to correct or delete personal data concerning him or her or to limit the processing by the data processor. Furthermore, a right of objection against the processing exists at any time. In the model this fact is taken into account by the concept dm:Approval which belongs to dm:Declaration.

Information must also be provided on whether and how automated decision-making, including profiling, takes place. According to Article 22 (1) and (4) of the GDPR (https://gdpr-info.eu/art-22-gdpr/), meaningful information on the logic involved, the significance and the intended impact of such processing for the data subject must be provided. This requirement is considered separately in the domain model via the concept $d m$ :DecisionMaking. It can be generated at runtime when system functionalities for decision making, such as a personalized recommendation (a subclass of $d m$ :Matching), is performed based on user data.

\section{Intelligibility}

The challenges of the comprehensibility of system processes also include their presentation and intelligibility. Users should be able to understand why something happens and how it happens. The representation of only technical information is not sufficient [7].

The formal modelling of conditions, requirements and declarations can support intelligibility and comprehensibility. The model can describe what has to be traced in the system by conditions and under what circumstances this should happen. Related to the GDPR example the data usage has to 
be made transparent, no matter whether it concerns the simple storage, the passing on or an automated processing to decision-making or profiling. At runtime, the system can be aware of which requirements apply through the concept $d m$ :Requirement and its linking to applications and application functionality. These relationships can be explained to the users.

In addition, intelligibility is facilitated by the deposit of target-group-specific texts (e.g. texts created by experts) and explanations through runtime integrated dictionaries.

\section{E. Supporting the Provision of Information on Data Usage}

APLE is an application which is linked to CONTact via context (Fig. 1) and provides the learning content. The CONTact platform contains the components for comprehensibility. The user's requests are forwarded to CONTact, which combines various applications, resources and functionalities. CONTact is responsible for the creation of the data usage explanation and initiates the retrieval of the information. APLE does not have to integrate the functionalities of the legal examination on its own, but shall provide the data stored about the user, its processing purpose and its use to the CONTact platform via an interface.

Our scenario takes place when the student Alice wants an overview of the stored and processed (personalized) data about her in the APLE environment.

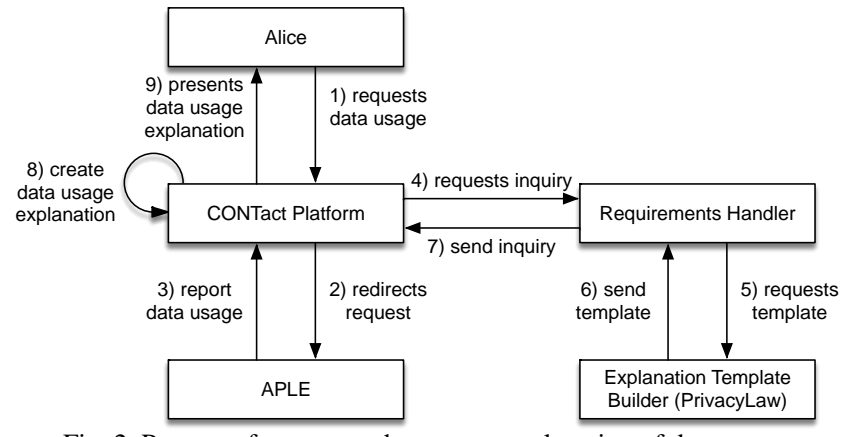

Fig. 2. Process of request and create an explanation of data usage.

Fig. 2 shows the process flow and the components involved, which leads to the presentation of an explanation of data usage to Alice.

Alice wants to know what data about her is stored and processed in the personalized learning environment APLE. For this she uses a function to request data information, which makes a request to CONTact (1). The comprehensibility components of CONTact get active and redirect the request to $A P L E(2)$.

The APLE application checks whether data is stored on Alice and for what purpose it is processed. APLE reports the result back to CONTact (3). Based on the returned data CONTact checks which conditions are affected and sends an inquiry (4) to the Requirements Handler. The handler determines that legal requirements are affected and requests (5) an appropriate explanation template.

The Explanation Template Builder creates a suitable template for the privacy law based on the available data and its processing. The template that specifies the legal claim or task of the system is (6) returned to the handler. The Requirements Handler returns (7) the inquiry results to
CONTact. It integrates the APLE report and creates an explanation of the data processing (8). After that, CONTact provides the explanation of data usage, e.g. in shape of a display window for Alice.

\section{DISCUSSION}

The presented domain model shows the connection between applications and legal requirements. The link to personalized, adaptive learning environments was only implicitly illustrated by APLE, which is an application that can be integrated into the context. Not part of the domain model is the comprehensibility and intelligible explanation of system processes and function calls in pedagogical and learner models. These still has to be researched.

Adaptive, personalized learning environments require adaptation rules. These are used to adapt the contents of the learning environment, its presentation and availability to the situation of the learner. Regarding to our four-layer context model [13], we modeled our domain model independently from adaptation rules. Nevertheless, the adaptation rules can also be explained to users through the concepts of the presented domain model.

\section{CONCLUSION}

We have presented a domain model in which external requirements for the use of systems are formally modeled. We have explained how the domain model can link the context of application and the context of a legal domain. The dependencies of the concepts dm:Condition, $d m$ :Requirement and dm:Declaration makes it possible to trace what and way something happen in the system.

The formal description was transferred into an ontology that can be used for the development of context-based systems. Based on this, it is possible to support users with intelligible and comprehensible explanations of system processes.

With an example we showed how legal requirements on privacy law and the right of access to information can be linked to a specific situation in an application.

\section{FUTURE WORK}

Future work will focus on extending the presented model with pedagogical aspects and learner concepts. As an extension, the domain model of [5] can be used. It contains a detailed domain description of pedagogical concepts and learner contexts. This will require a review of how the integration can work in order to link comprehensibility and the legal regulations. For this purpose, scenarios must be developed to test applicability in the field of personalized, adaptive learning environments.

In addition, appropriate adaptation rules must be developed for the domain of education, which can be applied at runtime on the basis of the domain model.

Our future work will also deal with the development of components for comprehensibility and intelligibility which shall be used in different domains. 


\section{CONFLICT OF INTEREST}

The authors declare no conflict of interest.

\section{AUTHOR CONTRIBUTIONS}

Mandy Goram and Dirk Veiel jointly wrote the article. Mandy Goram designed the concepts and relationships of the formal domain model. Her contribution covers the linking of legal and domain-specific aspects of the domain and context model. Dirk Veiel's contribution includes the description of an exemplary process for interaction and integration of the APLE environment into the CONTact platform. All authors had approved the final version.

\section{ACKNOWLEDGEMENT}

This work was supported by the Research Cluster Digitalization, Diversity and Lifelong Learning. Consequences for Higher Education $\left(D^{2} L^{2}\right)$ of the FernUniversität in Hagen funded by the Ministry of Culture and Science of the German State of North Rhine-Westphalia.

\section{REFERENCES}

[1] M. Monova-Zheleva, "Adaptive learning in web-based educational environments," Cybernetics and Information Technologies, vol. 5, no. 1, pp. 44-55, 2005.

[2] D. Burgos, C. Tattersall, and R. Koper, "How to represent adaptation in e-learning with IMS learning design," Interactive Learning Environments, vol. 15, no. 2, pp. 161-170, 2007.

[3] P. Rosmalen, H. Vogten, R. Es, H. Passier, P. Poelmans, and R. Koper, "Authoring a full life cycle model in standards-based, adaptive e-learning," Journal of Educational Technology \& Society, vol. 9, no. 1, pp. $72-83,2006$

[4] A. K. Dey, "Understanding and using context," Personal and Ubiquitous Computing, vol. 5, no. 1, pp. 4-7, 2001.

[5] M. G. Abarca, R. A. Alarcon, R. Barria, and D. Fuller, "Context-based e-learning composition and adaptation," in Proc. OTM Confederated International Conferences on the Move to Meaningful Internet Systems, Springer, 2006, pp. 1976-1985.

[6] D. Veiel, J. M. Haake, S. Lukosch, and G. Kolfschoten, "On the acceptance of automatic facilitation in a context-adaptive group support system," in Proc. 2013 46th Hawaii International Conference on System Sciences, IEEE, 2013, pp. 509-518.

[7] A. K. Dey, "Explanations in context-aware systems," in Proc. the Fourth International Conference on Explanation-aware Computing (EXACT'09), 2009, pp. 84-93.

[8] N. Guarino, "Formal ontology in information systems," in Proc. the First International Conference (FOIS'98), IOS press, 1998, vol. 46, pp. $3-15$.
[9] M. Rani, R. Nayak, and O. P. Vyas, "An ontology-based adaptive personalized e-learning system, assisted by software agents on cloud storage," Knowledge-Based Systems, vol. 90, pp. 33-48, 2015.

[10] E. G. Poitras and S. P. Lajoie, "Developing an agent-based adaptive system for scaffolding self-regulated inquiry learning in history education," Educational Technology Research and Development, vol. 62, no. 3, pp. 335-366, 2014.

[11] M. Yaghmaie and A. Bahreininejad, "A context-aware adaptive learning system using agents," Expert Systems with Applications, vol. 38, no. 4, pp. 3280-3286, 2011.

[12] I. Ganchev, S. Stojanov, M. Odroma, and D. Meere, "Development of infostation-based and contextaware mlearning system architectures," Advanced Learning, IntechOpen, 2009, pp. 115-139.

[13] J. M. Haake, T. Hussein, B. Joop, S. Lukosch, D. Veiel, and J. Ziegler, "Modeling and exploiting context for adaptive collaboration," International Journal of Cooperative Information Systems, vol. 19, no. 01-02, pp. 71-120, 2010.

[14] S. S. Hussain, D. Veiel, J. M. Haake, and S. Lukosch, "Facilitating understanding of team-based adaptation policies," in Proc. 6th International Conference on Collaborative Computing: Networking, Applications and Worksharing (CollaborateCom 2010), IEEE, 2010, pp. 1-8.

[15] M. A. Musen, "The protégé project: A look back and a look forward. AI Matters," Association of Computing Machinery Specific Interest Group in Artificial Intelligence, vol. 1, no. 4, 2015.

Copyright (C) 2020 by the authors. This is an open access article distributed under the Creative Commons Attribution License which permits unrestricted use, distribution, and reproduction in any medium, provided the original work is properly cited (CC BY 4.0).

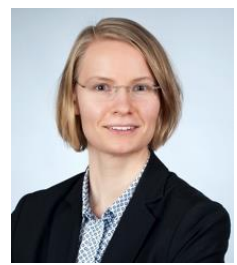

Mandy Goram holds an B.Sc. in business informatics and a M.Sc. in practical computer science from the FernUniversität in Hagen. She is a $\mathrm{PhD}$ candidate at the chair of cooperative systems at the Faculty of Mathematics and Computer Science of the FernUniversität in Hagen. Previously she has worked as head of business intelligence in a pharmaceutical company and as consultant for software development and data analytics in different branches. Her research interests include context-based intelligent software assistants, personalized adaptive environments, multi-agent systems, context-based data mining and compliance by design.

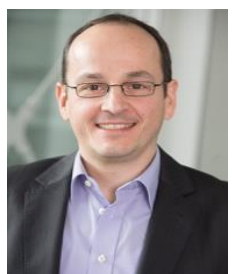

Dirk Veiel is a senior researcher at the chair of cooperative SYSTEMS at the Faculty of Mathematics and Computer Science of the FernUniversität in Hagen. He obtained his doctoral degree in computer science in 2013. His research interests include intelligent group/community support systems, context-based adaptation, community support systems for elderly, intelligent assistance systems, formal context representations, emergence in socio-technical systems, multi-agent systems, artificial intelligence and natural language processing. 